\title{
Avaliação de um Programa de Promoção de Competências de Literacia e
}

\author{
Numeracia

\section{Evaluation of a Literacy and Numeracy Competency Promotion Program}

\author{
Ana Rita Cardoso, Ana Rodrigues Costa, Diana Rocha \\ Universidade Fernando Pessoa
}

\begin{abstract}
Resumo
Neste trabalho foi desenvolvido e aplicado um programa de promoção de competências denominado "Do Brincar ao Saber" para avaliação do efeito do mesmo na evolução das competências de leitura, escrita e matemática. Na investigação participaram 52 crianças (5-6 anos), que frequentam o ensino pré-escolar, divididas em dois grupos (experimental e de comparação). O programa foi implementado ao longo de 16 sessões bissemanais e os dois grupos foram avaliados em dois momentos. Os resultados apontam para uma evolução mais acentuada ao nível das competências globais de aprendizagem no grupo experimental.

Palavras-chave: educação pré-escolar, competências de literacia emergente, competências matemáticas.
\end{abstract}

\begin{abstract}
In this work it was developed and implemented a competence developing programme called "Do Brincar ao Saber" (From Playing to Knowing) to evaluate the progamme effect in the evolution of the reading, writing and maths competences. Fifty-two children participated in this investigation (5-6 years-old), that are in the last grade of preschool. This programme was implemented during 16 sessions and each of the children were evaluated in two moments. The results point to a more pronounced evolution in the global learning competences in the experimental group.

Keywords: pre-school education, emerging literacy skills, mathematical skills.
\end{abstract}

\section{Literacia Emergente}

De acordo com diversos estudos (Leal, Peixoto, Silva, \& Cadima, 2006; Matosinhos, 2009; Santos, 2015), são de elevada importância as competências, conhecimentos e atitudes que a criança desenvolve em contexto pré-escolar e familiar para o processo de aquisição formal da numeracia e literacia que decorre mais tarde, assim como para todos os domínios da vida (Aguiar \& Leal, 2010).

A literacia emergente diz respeito aos conhecimentos, capacidades e atitudes precursores do desenvolvimento da linguagem escrita que precedem o seu ensino formal (Neuman \& Dickinson, 2001) e constitui-se como a primeira fase da construção e do desenvolvimento da literacia, permitindo a compreensão de muitas características e funções da linguagem e, por outro lado, a formação de conceitos e o desenvolvimento de capacidades específicas de leitura e de escrita (Gomes \& Santos, 2005)

O conceito de literacia emergente vem, então, reforçar a ideia de que o período pré-escolar desempenha um papel essencial no posterior processo de aprendizagem da leitura e da escrita (Leal, Peixoto, Silva, \& Cadima, 2006; Mata, 1999).

\section{Numeracia Emergente}

A matemática representa um domínio específico da Área de Expressão e Comunicação nas Orientações Curriculares para a Educação Pré-Escolar (Ministério da Educação, 1997) e é reconhecida como uma forma de comunicação, complementar à língua materna, com as suas próprias regras e simbolismo, que permite compreender e interpretar o mundo que nos rodeia (Pinto, 2011).

Segundo Barros e Palhares (1997) e Guerreiro (2014), cabe ao jardim de infância, o papel de organizar e consolidar os conhecimentos e capacidades espontaneamente desenvolvidos no dia-a-dia.

Para compreender os números é necessário possuir habilidades como a capacidade para classificar, ordenar, conhecer os diferentes tipos de ordem para poder contar, ter noção da organização espacial e saber coordenar e ter conhecimento de que os números são representados por signos convencionais (Somoza \& Portugal, 2011).

Para facilitar o desenvolvimento do conhecimento matemático, é essencial o desenvolvimento coeso da noção de número, formas, padrões, medidas e orientação espacial. As atividades realizadas devem apelar ao cotidiano das crianças e aos seus interesses, em particular, assim como estimular a curiosidade e motivá-las para a aprendizagem (Copley, 1999).

\section{Estudo Empírico}

A pergunta de partida à qual procuramos dar resposta foi a seguinte: Será que as competências necessárias à aquisição da leitura, da escrita e da matemática melhoram com a frequência de um programa de intervenção nessas áreas?

Objetivos de Investigação 
O presente estudo tem como principais objetivos elaborar, implementar e avaliar a eficácia de um programa de promoção de competências de literacia e numeracia no pré-escolar, bem como contribuir para o desenvolvimento dessas mesmas competências nas crianças pré-escolares.

\section{Método}

Participantes. Participaram, neste estudo, 52 crianças, de 5 e 6 anos de idade, que frequentam o último ano do ensino pré-escolar de um Agrupamento de Escolas do grande Porto, distribuídas por dois grupos, o grupo experimental $(n=26)$ e o grupo de comparação $(n=26)$. Considerámos um Grupo de Comparação pela ausência de controlo de variáveis como a etnia e condições socioeconómicas, por exemplo (Almeida \& Freire, 2008).

O critério de seleção prendeu-se unicamente com o facto de estas irem frequentar o $1 .^{\circ}$ ciclo do ensino básico, assim como possuírem 5 e/ou 6 anos de idade, sendo estas as únicas variáveis controladas, pelo que se caracteriza como sendo uma amostra por conveniência.

Materiais. Foi utilizado, como instrumento de recolha de dados, o "Pré-escolar" (la Cruz, 1985), adaptado para a população portuguesa por Coelho e Remédio (1993), que é um instrumento de avaliação das aptidões básicas envolvidas na aprendizagem escolar e que tem como objetivo avaliar a maturidade da criança para o início da escolaridade.

Foi ainda construído e administrado o Programa "Do brincar ao saber" que se afigura como sendo um Programa de Desenvolvimento de Competências Linguísticas e Matemáticas, que pretende contribuir para o processo de aprendizagem da leitura, escrita e competências matemáticas das crianças pré-escolares.

Constituído por 13 sessões, de caráter bissemanal, com cerca de uma hora cada, tem como objetivos primordiais contribuir, ao nível da motivação, para a literacia e numeracia, incentivar ao desenvolvimento da consciência fonológica, do impresso, morfossintática e ainda numérica, propiciando a compreensão das relações entre a linguagem oral e escrita, entre os números e o que estes representam efetivamente e como se podem representar, promover o desenvolvimento da linguagem oral, bem como o conhecimento das letras, dos números e das formas, entre outras, permitindo, assim, trabalhar as concetualizações das crianças face à leitura, à escrita e às competências matemáticas (Almeida, 2011; Ginsburg, Greenes, \& Balfanz, 2012; Gomes \& Santos, 2005; Rodrigues, 2013; Santos, 2015; Viana, 2002).

Foi ainda elaborada a autorização para a Direção do Agrupamento e para os Encarregados de Educação, relativamente à participação dos educandos no Programa, bem como a declaração de consentimento informado e as fichas de avaliação de cada sessão e do programa em geral.

Procedimento. Iniciamos todo o processo pela realização de pesquisa bibliográfica como forma de compreender, enquadrar e contextualizar a temática.

De seguida, elaboramos um projeto de investigação que seguiu para a Comissão de Ética da Universidade
Fernando Pessoa com o intuito de solicitar um parecer e a sua aprovação.

Após esta etapa, para a aplicação do programa de intervenção, foi feito o pedido de autorização à Direção do Agrupamento, bem como dos jardins-de-infância selecionados.

No seguimento deste processo, demos início à seleção da amostra. Os jardins de infância foram selecionados em virtude da proximidade em termos geográficos, como forma de conciliar mais facilmente a administração do Programa e do pré e pós-teste.

Passamos, de seguida, à recolha das autorizações dos Encarregados de Educação e à assinatura do consentimento informado.

Posteriormente, sendo que o pré-teste e o pós-teste nos permitem avaliar a eficácia do programa de intervenção (Almeida, \& Freire, 2008), foi administrado às crianças o instrumento de avaliação, o "Pré-escolar", na primeira sessão antes do início do programa e também na última sessão do mesmo, dos dois grupos.

Imediatamente após, demos início à implementação do Programa. A distribuição das crianças pelos grupos não foi realizada de forma aleatória, pelo que estamos perante um design quase experimental (Almeida \& Freire, 2008). A cada criança foi atribuído um código, dada a necessidade de se efetuar uma segunda avaliação (pós-teste).

Assim, não se pode garantir a existência de um grupo de controlo, mas sim de comparação, o que foi tido em conta aquando da análise das diferenças verificadas entre os dois grupos, já que estas não puderam ser atribuídas apenas à aplicação do programa de intervenção (Almeida \& Freire, 2008).

A administração do programa decorreu ao longo do $3 .^{\circ}$ período, iniciando-se em abril de 2016. No final das sessões era preenchida a Ficha de Avaliação em grupo, pelas crianças, na qual, um menino por sessão, pintava a carinha que o grupo escolhia para descrever a sessão. Após este procedimento, cada criança preenchia a sua ficha individual com um desenho do que mais e menos gostaram durante a sessão.

Antes mesmo de iniciar o Programa, foram realizadas reuniões com as Educadoras e, ainda, com os Encarregados de Educação, com vista à apresentação do Programa.

Este procedimento repetiu-se após a conclusão das sessões para devolução dos resultados obtidos.

\section{Resultados}

Com vista a avaliar a eficácia do Programa, verificando se existem diferenças entre o grupo de comparação e o grupo experimental no primeiro e segundo momentos, procedeu-se a uma análise descritiva dos resultados obtidos na prova "Pré-escolar". Assim, de uma forma geral, é possível compreender que os resultados obtidos se encontram entre valores mínimos e máximos esperados para cada subprova.

De modo a verificar as diferenças entre o primeiro e o segundo momento de avaliação, tanto no grupo experimental como no de comparação e, assim, avaliar a eficácia do Programa, comparando as médias obtidas 
entre os dois grupos, Experimental e de Comparação, efetuou-se uma análise com base no Teste de Mann-Whitney.

Então, foi possível analisar que não se verificam diferenças significativas entre os dois grupos nos dois momentos de avaliação.

No entanto, no momento do pós-teste, pela análise das médias obtidas na prova "Pré-escolar", na globalidade da prova administrada, a pontuação total obtida situa-se, mais uma vez, acima do esperado para indivíduos nesta faixa etária, em ambos os grupos, sendo que a média mais elevada pertence ao Grupo Experimental.

Foi ainda analisada a evolução dos dois grupos do primeiro para o segundo momento e procedeu-se à realização do teste de Wilcoxon, com um nível de significância de 0,05. Foi também elaborado um gráfico, relativo às médias dos resultados, através do qual pode ser observado o declive que caracteriza a evolução dos resultados entre o primeiro e o segundo momento de avaliação em cada um dos grupos.

Assim, apurou-se que existe uma diferença significativa entre os resultados do grupo experimental nos dois momentos de avaliação $(Z=-3,146, p=0,002)$. A diferença dos resultados obtidos pelo grupo de comparação nos dois momentos não é considerada significativa $(Z=-0,786, p=0,432)$. Os dados da Figura 1 permitem verificar que ocorreram evoluções no grupo experimental e no de comparação, entre o primeiro e o segundo momento de avaliação. $O$ declive mais acentuado da reta do grupo experimental indica que a evolução foi mais acentuada neste grupo.

Para além disso, através da avaliação do Programa efetuada pelas crianças, sessão a sessão, foi possível apurar que todo o grupo $(100 \%)$ selecionou a carinha verde como a mais indicada para descrever a sessão.

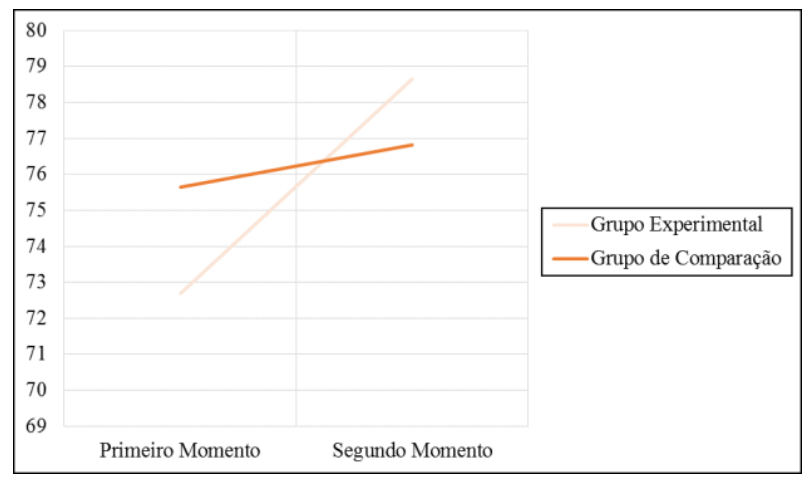

Figura 1. Valores médios do grupo de controlo e do grupo experimental na prova "Pré-escolar", no primeiro e segundo momentos de avaliação

\section{Discussão dos Resultados}

De acordo com os resultados observados, estão reunidos os elementos para traçar uma análise crítica e sintetizada, sustentada na teoria que fundamenta o presente estudo.

Desta forma, o objetivo principal deste trabalho consistia em elaborar, implementar e avaliar a eficácia de um programa de promoção de competências de literacia e numeracia no pré-escolar, sendo que os resultados apresentados sustentam a eficácia do programa.

Assim, é possível concluir que se atingiram, de forma positiva, os objetivos, já que se verifica uma evolução significativa no desempenho das crianças do grupo experimental do primeiro para o segundo momento de avaliação. De facto, se compararmos crianças que foram submetidas ao programa de intervenção (grupo experimental) com crianças que não foram submetidas a qualquer programa (grupo de comparação), verificamos que se registou uma evolução mais acentuada no primeiro grupo de crianças.

Porém, essas diferenças não são estatisticamente significativas, pelo que se pode interpretar que o número reduzido de elementos da amostra possa condicionar este aspeto. No entanto, segundo Martins (2011), não é incorreto recorrermos à análise das médias para analisarmos os grupos, mesmo utilizando o teste Mann-Whitney para avaliar as diferenças entre grupos e, pela análise das médias obtidas pelos dois grupos no segundo momento de avaliação, é possível apurar que se verifica um valor superior e uma evolução maior no Grupo Experimental. Ainda assim, foi possível constatar que se verificaram diferenças estatisticamente significativas nos grupos face aos dois momentos de avaliação, tendo-se verificado uma evolução positiva do primeiro para o segundo momento.

Por outro turno, o Grupo de Comparação, aquando do primeiro momento de avaliação já apresentava resultados superiores ao Grupo Experimental, sendo que este último apresenta uma evolução muito maior em termos de médias.

Assim, estes resultados são reforçados pela literatura e vão ao encontro do defendido e comprovado por Alves (2010), Azevedo (2011), Cruz, et al. (2008), Guerreiro (2014), Mata (2012), Matosinhos (2009), Rodrigues (2003), Santos (2015), Silva (2013) e estão, também, em consonância com o estipulado pelas Orientações Curriculares para a Educação Pré-Escolar (1997), que defendem este tipo de intervenções parece promover um melhor desempenho das competências trabalhadas nas crianças envolvidas.

Verificaram-se ainda ganhos ao nível da escrita, pelo facto de terem sido desenvolvidas, analogamente, atividades de escrita inventada e de consciência fonológica. Tal como analisado por Silva (2003), esta é uma ferramenta essencial que permite à criança a tomada de consciência, a compreensão e apreensão autónoma da existência de pequenas unidades sonoras. Ao longo das sessões foi notório que os conhecimentos como a consciência fonológica, o nome e som das letras e ainda a sua representação gráfica, foram evoluindo no que respeita às escritas inventadas, estando este facto de acordo com a literatura (Silva, 2003).

Também foi possível constatar que se verificaram diferenças significativas nos grupos face aos dois momentos de avaliação, tendo-se verificado uma evolução positiva do primeiro para o segundo momento.

Importa destacar que não foi possível, durante a administração do Programa, controlar a origem étnica dos participantes e, assim, a composição dos grupos desta investigação confirma alguns estudos que associam 
características desenvolvimentais e dos contextos familiares com as competências demonstradas pelas crianças no primeiro momento de avaliação.

As crianças do grupo de comparação pertencem a níveis sócioeconómicos mais favorecidos. Segundo Leal e colaboradores (2005), famílias provenientes de meios socialmente desfavorecidos tendem a dar menos importância aos aspetos da literacia, providenciando, portanto, aos seus filhos menos oportunidades para o desenvolvimento deste tipo de competências. Peixoto e colaboradores (2008), por sua vez, relatam que crianças que vivem em ambientes de baixos recursos e/ou com um dos pais com baixa escolaridade têm menos oportunidades para desenvolver competências de literacia.

Neste sentido, os objetivos e os resultados do presente estudo encontram-se fundamentados empiricamente, já que a intervenção foi implementada com crianças em risco de desenvolvimento ao nível deste tipo de competências, tendo conseguido atingir resultados positivos.

O presente programa teve também a particularidade de ser em grupo, podendo justificar, desta forma, os maiores benefícios retirados pelas crianças do grupo experimental, uma vez que, à semelhança de investigações como as de Teberosky (1987), Silva (1992) Mata (1995), Lima (2012) e Gonçalves (2012) também nos foi possível constatar que as interações entre pares são impulsoras de progressos.

\section{Referencias}

Aguiar, T., \& Leal, T. (2010). Competências de Literacia, de Numeracia e Sociais em Dois Períodos do Desenvolvimento da Criança: no Pré $\operatorname{escolar}$ e no $1^{\circ}$ Ano de Escolaridade. Revista Galego-Portuguesa de Psicoloxía e Educación, 18(2), 1138-1663.

Almeida, S. (2011). “A ler bem vou mais além”: Estudo da relação entre as competências leitoras e pré-leitoras e a obtenção de melhores resultados a Língua Portuguesa no $1^{\circ}$ ano de escolaridade (Dissertação de Mestrado). Disponível em: http://hdl.handle.net/10284/1985 .

Almeida, L., \& Freire, T. (2008). Metodologia da investigação em Psicologia e Educação. (5 Edição). Braga: Psiquilíbrios.

Alves, A. (2010). Intervenção nas competências de consciência fonológica num grupo de crianças em risco: O programa "Era uma vez...o mundo dos sons!" (Dissertação de Mestrado). Universidade do Porto, Porto.

Aragón, E., Navarro, J., Aguilar, M. \& Cerda, G. (2015). Predictores cognitivos del conocimiento numérico temprano en alumnado de 5 años. Revista de Psicodidáctica, 20(1), 83-97.

Baroody, A. (2000). El pensamento matemático de los niños - Un marco evolutivo para maestros de preescolar, ciclo inicial y educación especial. Madrid: Visor Dis.

Barros, M. \& Palhares, P. (1997). Emergência da matemática no jardim-de-infância. Porto: Porto Editora.
Betts, J., Pickart, M. \& Heistad, D. (2009). Construct and predictive validity evidence for curriculum-based measures of early literacy and numeracy skills in kindergarten. Journal of Psychoeducational Assessment, 27(2), 83-95.

Copley, J. (1999). Mathematics in the early years. (1. ${ }^{\mathrm{a}}$ edição). USA: The National Council of Teachers of Mathematics.

Dalfovo, M., Lana, R., \& Silveira, A. (2008). Métodos quantitativos e qualitativos: um resgate teórico. Revista Interdisciplinar Científica Aplicada, 2(4), 01-13.

Educação, M. (1997). Orientações Curriculares para a Educação Pré-escolar. Lisboa: Editorial do Ministério da Educação.

Ginsburg, H., Greenes, C., \& Balfanz, R. (2012). Brincando com a Matemática: Programa de Desenvolvimento de Competências Matemáticas em Idade Pré-escolar. (1. a edição.

Gomes, I., Santos, N. (2005). Literacia Emergente: “É de pequenino que se torce o pepino!". Revista da Faculdade de Ciências Humanas e Sociais, 2, 312-326.

Gonçalves, L. (2012). Contributo Para A Avaliação Da Leitura Conjunta Em Crianças Em Idade Pré-Escolar (Dissertação de Mestrado). Retirado de: http://catalogo.up.pt/exlibris/aleph/a18_1/apache_med ia/L5AFDDQK37SA7MD7FDV6XQ5EKDUJEK.pdf

Guerreiro, M. (2014). Padrões de Gustav Klimt: Uma Experiência Matemática no Pré-escolar (Dissertação de Mestrado). Retirado de: http://hdl.handle.net/10400.1/8221 .

Leal, T., Cadima, J., Silva, P. \& Gamelas, A. M. (2005). A aprendizagem da leitura e da escrita ao longo do $1^{\circ}$ ciclo E.B.: Contributos de um programa de literacia numa comunidade em desvantagem social. In J. Bairrão (Org.), Desenvolvimento: Contextos Familiares e Educativos (256-282). Porto: Livpsic.

Leal, T., Peixoto, C., Silva, M., \& Cadima, J. (2006). Desenvolvimento da literacia emergente: Competências em crianças de idade pré-escolar. Actas do $6^{\circ}$ Encontro Nacional $\left(4^{\circ}\right.$ Internacional $)$ de Investigação em leitura, Literatura Infantil e Ilustração. Braga: Universidade do Minho.

Lima, M. (2012). As Crianças e a Aprendizagem da Escrita e da Leitura: Impacto de um Programa de Escrita Inventada em Situação de Grupo. (Dissertação de Mestrado). ISPA, Lisboa.

Lopez, E., Gallimore, R., Garnier, H. \& Reese, L. (2007). Preschool antecedents of mathematics achievement of latinos - The influence of family resources, early literacy experiences, and preschool attendance. Hispanic Journal of Behavioral Sciences, 29(4), 456-471.

Martins, C. (2011). Manual de Análise de Dados Quantitativos com Recurso ao IBM SPSS: Saber Decidir, Fazer, Interpretar e Redigir. (1. ${ }^{\mathrm{a}}$ edição). Braga: Psiquilíbrios Edições.

Mata, L. (1995). Escrita em interacção: Processos de construção em crianças de 5-6 anos. (Dissertação de Mestrado). ISPA, Lisboa. 
Mata, L. (1999). Literacia - O papel da família na sua apreensão. Análise Psicológica, 1(17), 65-77.

Mata, S. (2012). O Ensino da Matemática na Educação Pré-Escolar e no Ensino do $1^{\circ}$ Ciclo do Ensino Básico.

Matosinhos, C. (2009). A Ler Vamos... - Intervenção Precoce na Aprendizagem da Leitura e Escrita. Câmara Municipal de Matosinhos: Divisão de Educação e Formação.

Neuman, S., \& Dickinson, D. (2001). Handbook of Early Literacy Research. (1. ${ }^{\mathrm{a}}$ edição). Nova Iorque:The Guilford Press.

Peixoto, C., Leal, T., \& Cadima, J. (2008). Comportamentos Interactivos de Leitura Conjunta Adulto-Criança. Retirado de: http://www.casadaleitura.org/portalbeta/bo/document os/ot_comportamentos_interactivos_leitura_conjunta_ b.pdf.

Rodrigues, J. (2013). Avaliação do Impacto de um Programa de Intervenção de Sensibilização à Linguagem Escrita (Dissertação de Mestrado). Disponível em: http://hdl.handle.net/10400.12/2769 .

Santos, V. (2015). Análise da Influência Reciproca entre Competências Matemáticas e Competências de Literacia Emergente na Educação Pré-escolar (Dissertação de Mestrado). Disponível em: http://hdl.handle.net/10284/4810 .

Silva, A. (1992). Estudo comparativo dos processos interactivos numa tarefa de escrita em crianças com competências fonológicas diferenciadas. (Dissertação de Mestrado).

Silva, A. (2003). Até à descoberta do princípio alfabético. Coimbra: Fundação Calouste Gulbenkian.

Somoza, S. \& Portugal, M. (2011). Competencias numéricas de los niños/as al comenzar la educación infantil. Investigación en Educación Matemática. Comunicaciones de los Grupos de Investigación de la SEIEM, 439-451. Lleida.

Teberosky, A. (1987). Os processos de leitura e escrita. Porto Alegre: Artes Médicas.

Viana, F. (2002). Melhor Falar para Melhor Ler. Um Programa de Desenvolvimento de Competencias Linguisticas (4-6 anos). (2. edição). Minho: Centro de Estudos da Criança - Universidade do Minho.

Viana, F., \& Teixeira, M. (2002). Aprender a ler - da aprendizagem informal à aprendizagem formal $\left(1 .^{\mathrm{a}}\right.$ edição). Porto: ASA Editores. 